\title{
Protocolo de Avaliação de Crianças com Autismo: Evidências de Validade de Critério
}

\author{
Daniela Fernandes Marques ${ }^{1}$ \\ Cleonice Alves Bosa \\ Universidade Federal do Rio Grande do Sul
}

\begin{abstract}
RESUMO - A avaliação de crianças com suspeita de Transtornos do Espectro do Autismo (TEA), na saúde pública, demanda instrumentos construídos e validados no Brasil. O objetivo deste estudo é verificar evidências de validade de critério, preliminares, do Protocolo de Avaliação para Crianças com Suspeita de Transtornos do Espectro do Autismo (PRO-TEA). Participaram 30 crianças, entre dois e quatro anos, divididas em três grupos (Desenvolvimento Típico, Síndrome de Down e Autismo). Os itens do PRO-TEA foram examinados por dois observadores independentes, cegos ao diagnóstico das crianças. A fidedignidade entre os avaliadores foi examinada por meio do coeficiente Kappa. Os itens referentes à atenção compartilhada, brincadeira simbólica e movimentos repetitivos do corpo discriminaram o grupo com TEA dos controles, demonstrando o potencial do instrumento.
\end{abstract}

Palavras-chave: transtorno autista, avaliação psicológica, validação, atenção compartilhada

\section{Evaluation Protocol for Children with Autism: Evidence of Criterion Validity}

\begin{abstract}
Evaluating children suspected of having Autism Spectrum Disorders (ASD) in public health departments of Brazil demands tools created and validated in that country. This study aimed to investigate the preliminar criterion validity of the Protocolo de Observação para Crianças com Suspeita de Transtornos do Espectro do Autismo (PRO-TEA). Thirty children, ages of two to four years old, were divided in three groups (Typical Development, Down syndrome and Autism). The items were examined by 2 independent observers, both unaware of the children's diagnosis. The reliability of the evaluators was examined with the Kappa coefficient. The items referring to joint attention, symbolic play and repetitive movements of the body discriminated the group with ASD from the control group, are an indication of the potential value of the instrument.
\end{abstract}

Keywords: autism, psychological evaluation, validation studies, joint attention

A terminologia "Transtorno do Espectro do Autismo" (TEA), de acordo com o DSM-5 (American Psychiatric Association [APA], 2013), designa uma condição neurodesenvolvimental que acomete uma em cada 88 crianças (Centers for Disease Control and Prevention, 2012). Tal condição é caracterizada por comprometimentos sociocomunicativos e pela presença de comportamentos repetitivos e estereotipados, independente da etiologia ou dos déficits associados (Rapin \& Tuchman, 2009).

$\mathrm{Na}$ área sociocomunicativa, comprometimentos na habilidade de atenção compartilhada (AC) têm sido considerados um forte sinal de alerta e um importante preditor de um eventual diagnóstico do transtorno do autismo (Charman, 2003; Dawson et al., 2004; Delinicolas \& Young, 2007). Definida pela ocorrência de comportamentos infantis com propósito declarativo, a AC envolve o contato visual coordenado com gestos e verbalizações para dividir a experiência em relação às propriedades dos objetos/eventos ao redor (Mundy \& Sigman, 1989).

Além da AC, também são considerados eventuais indicadores precoces do TEA a capacidade de imitar e de

1 Endereço para correspondência: Universidade Federal do Rio Grande do Sul, Instituto de Psicologia, Rua Ramiro Barcelos 2600, Santa Cecília, Porto Alegre, RS, Brasil. CEP: 90035003.E-mail: dmarques@hcpa. ufrgs.br brincar simbolicamente. A imitação, especialmente a que envolve símbolos linguísticos, proporciona aprendizagem acerca das ações e intenções do outro, bem como o compartilhamento de experiências e o senso de reciprocidade com um parceiro social (Meltzoff, 2005; Tomasello, Carpenter, Call, \& Moll, 2005). Por sua vez, a brincadeira simbólica, sobretudo a que envolve o uso de um objeto como um substituto de outro, apresenta-se limitada tanto em frequência quanto em variedade (Lifter, 2009), ocorrendo a predominância da brincadeira exploratória e/ou funcional (Blanc, Adrien, Roux, \& Bartélémy, 2005; Rutherford, Young, Hepburn, \& Rogers, 2007).

Por fim, na área da flexibilidade comportamental, a ocorrência de comportamentos repetitivos complexos (interesses restritos, ecolalia, insistência na mesmice e rituais elaborados) é considerada outro possível indicativo de TEA. Cabe ressaltar que os comportamentos repetitivos simples (movimentos estereotipados, tiques, manipulação repetitiva de objetos e formas repetitivas de comportamento automutilatório) podem ser encontrados em outras condições, como deficiência mental ou sensorial (Moraes, 2004).

O diagnóstico dos casos suspeitos de TEA pode ser realizado tanto com base na observação comportamental dos critérios dos sistemas de classificação quanto por meio do uso de instrumentos validados e fidedignos, que permitem ao profissional traçar um perfil refinado das características 
de desenvolvimento da criança. Na literatura internacional, figuram dois instrumentos considerados "padrão-ouro" para o diagnóstico: a Autism Diagnostic Interview-Revised (ADI-R) (Lord, Rutter, \& Le Couteur, 1994) e o Autism Diagnostic Observation Schedule-Generic (ADOS) (Lord, Rutter, Dilavore, \& Risi, 1999), ambos em fase inicial de validação no Brasil.

AADI-R é uma entrevista semiestruturada, administrada aos pais ou cuidadores e composta por 93 itens, divididos em seis seções (informações gerais sobre o paciente e sua família; desenvolvimento precoce e os marcos do desenvolvimento; tríade de comprometimentos segundo os critérios do DSMIV-TR; e problemas gerais de comportamento). O tempo previsto para sua administração por profissionais treinados e experientes é de aproximadamente 1,5 a 2,5 horas (Rutter, Le Couteur, \& Lord, 2003).

Já o ADOS-G é um instrumento padronizado e semiestruturado de observação que busca verificar especificamente as habilidades de interação social, comunicação, brincadeira e uso imaginativo de materiais pelas crianças com suspeita de TEA. É composto por quatro módulos que variam conforme os diferentes níveis de linguagem expressiva da criança. Tanto a ADI-R quanto o ADOS-G requerem um treinamento prévio na administração e codificação das respostas, que podem ser realizados com apoio de alguns centros no exterior. Mesmo após a validação, o amplo uso destes instrumentos somente pode ocorrer mediante a compra de direitos autorais da editora americana, por uma editora nacional. Na prática, isto significa que esses instrumentos não estão disponíveis para amplo e livre uso no Brasil, em curto prazo.

No que se refere aos instrumentos de triagem, tem-se no país alguns instrumentos adaptados e parcialmente validados: a Autistic Traits of Evaluation Scale (ATA) (Ballabriga, Escudé, \& Llaberia, 1994), por Assumpção Jr, Kuczynski, Gabriel e Rocca (1999); a Autism Behavior Checklist (ABC) (Krug, Arick, \& Almond, 1993), por Marteleto e Pedremônico (2005); a Childhood Autism Rating Scale (CARS) (Schopler, Reichler, \& Renner, 1988), por Pereira, Riesgo e Wagner (2008); e o Autism Screening Questionnaire (ASQ) (Berument, Rutter, Lord, Pickles, \& Bailey, 1999), por Sato et al. (2009). Além destes, foi realizada a tradução do instrumento Modified Checklist for Autism in Toddlers (M-CHAT) (Robins, Fein, Barton, \& Green, 2001) para o português brasileiro, por Losapio e Pondé (2008) e, posteriormente, parcialmente validada por Castro-Souza (2011).

Apesar da importância dos dados que os instrumentos internacionais nessa área têm gerado, há algumas dificuldades para sua utilização como: necessidade de treinamento de alto custo (caso dos instrumentos considerados "padrão-ouro"), categorias com amplas definições e predominantemente dicotômicas (comportamento/habilidade está ou não presente) e informação dependente dos cuidadores. Este último aspecto representa uma limitação em nossa cultura, devida principalmente à baixa escolaridade dos pais, por isso é importante que este tipo de fonte de informação seja combinada à observação direta da criança.

Outro aspecto fundamental é que há uma carência de instrumentos de observação do comportamento social e da brincadeira, que possam ser usados na rede pública de saúde. É importante que esses sejam de baixo custo, de rápida aplicação e treinamento e que possam ser administrados por profissionais de diferentes áreas da saúde. A observação direta do comportamento deve, sobretudo, levar em consideração as sutilezas da expressão dos comportamentos de crianças com autismo, isto é, não apenas a ocorrência ou não de um dado comportamento, mas sua intensidade, duração e peculiaridade. Embora o TEA seja caracterizado por um desvio qualitativo do desenvolvimento, é justamente a qualidade do comportamento que tende a perder relevo nas avaliações. Além disso, a evolução dos comportamentos tende a ser sutil e lenta, em muitos casos. Instrumentos com respostas amplas e dicotômicas não permitem o registro de avanços mais sutis, que tendem a ser pouco perceptíveis. Essa situação pode produzir resultados desalentadores, do ponto de vista do desenvolvimento, nas situações de reavaliações da criança, caso os instrumentos não consigam captar estes detalhes.

O Protocolo de Avaliação para Crianças com Suspeita de Transtornos do Espectro do Autismo (PRO-TEA) foi idealizado pelo Núcleo de Estudos e Pesquisas em Transtornos do Desenvolvimento - NIEPED/UFRGS, em 1998, e aprimorado em 2007. Surgiu em decorrência da necessidade de sistematizar a observação clínica em avaliações e reavaliações de crianças com suspeita de autismo, na ausência de instrumentos internacionais validados. $\mathrm{Na}$ prática, o PRO-TEA já vem sendo utilizado na clínica por diferentes especialistas (psicólogos, fonoaudiólogos, terapeutas ocupacionais), há cerca de uma década e em diferentes regiões do país, apontando para a urgência no exame de suas propriedades psicométricas. Seus itens foram gerados com base nos principais resultados da tese de doutorado da autora (Bosa, 1998) e operacionalizam as áreas que definiam o diagnóstico de autismo no DSMIV-TR. Os itens que compõem o bloco de interação social recíproca e brincadeira, fundamentaram-se na teoria do desenvolvimento sociopragmático (Tomasello \& Farrar, 1986) e foram integrados com os principais achados na área específica do autismo (Charman, 1997; Sigman, Mundy, Sherman, \& Ungerer, 1986).

O instrumento é de rápida aplicação por meio de observação direta da criança em interação com um adulto (pais e/ou profissional) e requer um conjunto de brinquedos de baixo custo e fácil reposição. Os itens do protocolo contemplam a tríade de comprometimentos, levando em consideração a frequência, intensidade e peculiaridade dos sintomas, assim como registros qualitativos, a partir de resultados de pesquisas na área. Contudo, havia uma necessidade de refinamento deste instrumento, tanto em termos de readequação de itens que se mostravam ambíguos quanto de busca de evidências de validade, examinando-se em que medida seus itens identificavam peculiaridades dos comportamentos do espectro, que distinguiriam estas crianças de outros grupos (e.g.: atraso de desenvolvimento).

Deste modo, o objetivo principal deste estudo foi examinar evidências de validade de critério preliminares do "Protocolo para Crianças com Suspeita de Transtornos do Espectro do Autismo". Mais especificamente, foi investigada sua propriedade discriminativa, por meio da verificação dos 
itens que mais distinguiram o grupo de crianças com TEA dos grupos controles. A hipótese era de que os itens que avaliavam imitação espontânea, atenção compartilhada, brincadeira simbólica e comportamentos repetitivos estariam mais comprometidos nas crianças com TEA, comparadas aos controles.

\section{Método}

\section{Delineamento e Participantes}

Neste estudo foi utilizado um banco de dados originário do estudo de Sanini (2006) ${ }^{1}$ cujo delineamento, quaseexperimental, era composto por três grupos contrastantes. O grupo 1 foi composto por crianças com autismo (AU), o grupo 2 por crianças com síndrome de Down (SD) e, finalmente, o grupo 3 por crianças com desenvolvimento típico (DT).

O grupo AU foi composto por 10 crianças, do sexo masculino, com diagnóstico de autismo realizado por especialistas com base nos critérios do DSM-IV-TR (American Psychiatric Association [APA] ,2002), conforme registros de clínicas e escolas especiais, escolhidas por critérios de conveniência. Foram selecionados apenas meninos pelo fato da prevalência ser quatro vezes maior em meninos do que em meninas (Centers for Disease Control and Prevention, 2012). As crianças tinham entre três e oito anos $(M=4,96$ anos; $S D=4,12)$. Não foram incluídos os casos de crianças com autismo ou com $S D$ que possuíam qualquer tipo de deficiência física ou sensorial associada, conforme registros profissionais. O grupo $S D$ foi composto por 10 crianças, do sexo masculino, equiparadas pela idade cronológica com as crianças do grupo AU, com idades entre 4 e 8 anos $(M=6,82$ anos; $S D=3,62)$, selecionadas por conveniência, com base nos registros de escolas de educação especial. O grupo DT foi composto também por 10 crianças do sexo masculino, com idades entre 1 e 3 anos $(M=2,62$; $S D=1,64)$, selecionadas em escolas de educação infantil, também por conveniência.

As crianças dos três grupos foram equiparadas em relação ao sexo e ao nível de linguagem verbal compreensiva e expressiva, com base no Inventário de Palavras (ver Di Napoli \& Bosa, 2005). Não foram utilizadas medidas de QI ou de idade mental nas crianças para fins de pareamento da amostra. Na tentativa de lidar com esta limitação, optou-se por selecionar as crianças dos grupos clínicos com no mínimo o dobro da idade cronológica em relação às crianças do grupo controle, visto que 60 a $70 \%$ das crianças com autismo apresentam deficiência mental (Klin, 2006).

\section{Instrumentos}

\section{Protocolo de Avaliação para Crianças com Suspeita de Transtornos do Espectro do Autismo - PRO-TEA}

Esse instrumento avalia tanto quantitativamente quanto qualitativamente a tríade de comprometimentos característicos do TEA em crianças em idade pré-escolar.
Dessa forma, consiste de três principais dimensões: Interação Social, Linguagem e Comunicação; Relação com os Objetos e Brincadeira; e, por fim, Comportamento Estereotipado e Autolesivo. Cada uma das dimensões é subdividida em itens que contemplam comportamentos característicos, reportados no DSM-IV-TR.

Adimensão "Interação Social, Linguagem e Comunicação" possui 14 itens: Saudação e Despedida; Atenção Compartilhada; Busca de Assistência; Responsividade Social; Imitação; Expressões Afetivas (Sorriso, Gama de Expressões), Reação à Imagem no Espelho; Produção de Palavras Espontâneas; Clareza; Qualidade; Habilidade ou Tentativas de "Conversar"; Compreensão de Palavras; Ecolalia e Rituais Verbais; e Comportamentos de Apego. A dimensão "Relação com os Objetos e Brincadeiras" possui 8 itens: Manipulação e Exploração; Formas de Exploração; Brincadeira Funcional; Brincadeira Simbólica; Sequência de Tópicos na Brincadeira Simbólica; Qualidade Representacional; Atividade Gráfica; e Qualidade da Representação. Por fim, a dimensão "Comportamento Estereotipado e Autolesivo" possui 4 itens: Movimentos Repetitivos das Mãos; Movimentos Repetitivos do Corpo; Autolesão; e Reação a Tentativas do Adulto de Interromper a Autolesão. Os itens do protocolo que focam especificamente a linguagem oral, os comportamentos de apego, a reação à imagem no espelho e os comportamentos autolesivos não foram examinados neste estudo.

As respostas são alocadas em uma escala de 3 pontos, que descrevem os comportamentos da criança, de acordo com a sua frequência e intensidade. A pontuação 1 significa melhor habilidade em uma determinada variável e 3, a pior. Os itens referentes à linguagem verbal têm o formato checklist, sendo assinaladas as características que aparecem na fala da criança. Finalmente, por se tratar de um contexto de observação naturalístico (livre) é levado em consideração se o comportamento está ausente por falha da criança ou por contingências do contexto (não houve oportunidade para que um determinado comportamento aparecesse; adulto não fez solicitações etc.). Esta opção de resposta está identificada nos itens pelo número 9 .

Foi elaborado um manual contendo a definição operacional dos comportamentos investigados, bem como as instruções quanto à administração e aferição do protocolo.

\section{Procedimentos}

\section{Procedimentos para Codificação do Protocolo}

A codificação dos itens foi realizada, de forma independente, pela pesquisadora responsável por este estudo e por uma aluna de pós-graduação strictu sensu, ambas cegas ao diagnóstico das crianças. A segunda avaliadora desconhecia os objetivos do estudo e foi treinada para realização dessa tarefa. O manual com orientações foi utilizado para fins de padronização dos registros.

1 Projeto aprovado pelo Comitê de Ética da UFRGS sob numero 2004356 
O cálculo de fidedignidade entre os avaliadores foi realizado por meio do coeficiente Kappa. Por tratar-se de variáveis categóricas, foram analisadas uma a uma. Para uma interpretação dos valores Kappa, foi utilizada as faixas de valores sugeridas por Altman $(1991)^{2}$.

$\mathrm{Na}$ análise de fidedignidade das variáveis, o item Movimento das Mãos revelou uma concordância moderada $(k=0,582 ; p<0,001)$. Os itens que apresentaram concordância boa foram Brincadeira Simbólica $(k=0,768 ; p<0,001)$, Sequência de Tópicos da Brincadeira Simbólica $(k=0,779$; $p<0,001)$, Qualidade da Representação da Brincadeira Simbólica $(k=0,737 ; p<0,001)$ e Movimentos do Corpo $(k=0,793 ; p<0,001)$. Por fim, a força de concordância muito boa foi encontrada nos itens Atenção Compartilhada $(k=0,925 ; p<0,001)$, Busca de Assistência $(k=0,830$; $p<0,001)$, Responsividade Social $(k=0,938 ; p<0,001)$, Imitação $(k=1,00 ; p<0,001)$, Sorriso $(k=0,843 ; p<0,001)$, Manipulação/Exploração $(k=0,815 ; p<0,001)$, Formas de Exploração $(k=0,939 ; p<0,001)$ e Brincadeira Funcional $(k=0,930 ; p<0,001)$. Dessa forma, os valores de concordância encontrados foram considerados satisfatórios no campo da Psicologia, incluindo os moderados. Não foram encontrados níveis de concordância pobre ou fraca.

Para o registro final, os itens em que as avaliadoras registraram respostas discordantes ${ }^{3}$, foram submetidos a um terceiro julgamento por um expert no assunto, que definiu a resposta mais adequada. Esse procedimento foi realizado para fins de determinação dos dados a serem analisados estatisticamente.

\section{Resultados}

A entrada e análise dos dados foi realizada utilizando o Statistical Package for the Social Sciences (SPSS), versão 17.0 e o Programs for Epidemiologists (PEPI), versão 4.0. As diferenças estatísticas significativas entre os grupos foi examinada por meio do teste não-paramétrico Kruskal-Wallis, em razão do tamanho da amostra e do tipo de variáveis (categóricas ordinais). Para investigar as diferenças entre dois grupos específicos (AU/SD, AU/DT, SD/DT) nos itens do protocolo, optou-se pelo teste Mann-Whitney. O teste Finner foi utilizado para o ajuste do nível descritivo amostral (valor- $p$ ). O teste Qui-quadrado foi utilizado no intuito de analisar as associações entre os diferentes grupos e os escores para cada item do protocolo (e.g.: se o desempenho do grupo com Autismo no item de Atenção Compartilhada está associado à opção de escore 1, 2 ou 3). O nível de significância adotado para todos os testes estatísticos foi de $5 \%(\mathrm{p} \leq 0,05)$.

A tabela 1 mostra que o grupo AU se diferenciou significativamente dos demais grupos nos itens Atenção Compartilhada (teste Kruskal-Wallis; $\chi^{2}=18,0 ; g l=2$; $\mathrm{p}<0,001$ ), Responsividade Social (teste Kruskal-Wallis; $\left.\chi^{2}=13,9 ; g l=2 ; \mathrm{p}=0,001\right)$, Brincadeira Simbólica (teste Kruskal-Wallis; $\chi^{2}=16,5 ; g l=2 ; \mathrm{p}<0,001$ ) e Movimentos do Corpo (teste Kruskal-Wallis; $\chi^{2}=17,7 ; g l=2 ; p<0,001$ ). Nos demais itens, o grupo AU se diferenciou apenas do grupo DT, não permitindo concluir se esta diferença se deve ao diagnóstico de autismo ou ao atraso de desenvolvimento também presente no grupo SD. Somente os itens em que

Tabela 1. Medianas, Percentil Inter-quartílico e Nivel de Significância dos Itens do PRO-TEA entre os grupos AU, SD e DT

\begin{tabular}{|c|c|c|c|c|}
\hline Variáveis/Item & $\begin{array}{c}\text { Grupo AU } \\
M d(P 25-P 75)\end{array}$ & $\begin{array}{c}\text { Grupo SD } \\
M d(P 25-P 75)\end{array}$ & $\begin{array}{c}\text { Grupo DT } \\
M d(P 25-P 75)\end{array}$ & $p^{*}$ \\
\hline Atenção compartilhada & $2,5(1,8-3,0)^{\mathrm{b}}$ & $1(1-1)^{\mathrm{a}}$ & $1(1-1)^{\mathrm{a}}$ & $<0,001$ \\
\hline Busca de assistência & $3,0(1,8-3,0)^{\mathrm{b}}$ & $1(1-2,5)^{\mathrm{a}, \mathrm{b}}$ & $1(1-1)^{\mathrm{a}}$ & $\mathbf{0 , 0 0 4}$ \\
\hline Responsividade social & $2(1,8-2,3)^{b}$ & $1(1-1)^{\mathrm{a}}$ & $1(1-1)^{\mathrm{a}}$ & $\mathbf{0 , 0 0 1}$ \\
\hline Imitação & $2(2-2)$ & $1,5(1-2)$ & $1(1-2)$ & $\mathbf{0 , 0 1 4} 4^{\dagger}$ \\
\hline Sorriso & $2(1-2,8)$ & $1(1-1)$ & $1(1-1)$ & $\mathbf{0 , 0 0 1}{ }^{\dagger}$ \\
\hline Manipulação/exploração & $2(1-2)$ & $1(1-1)$ & $1(1-1)$ & $0,016^{\dagger}$ \\
\hline Formas de exploração & $2(1,8-3)^{b}$ & $1(1-2)^{a, b}$ & $1(1-1)^{\mathrm{a}}$ & 0,001 \\
\hline Brincadeira funcional & $2(1,8-2)^{b}$ & $1(1-2)^{\mathrm{a}, \mathrm{b}}$ & $1(1-1)^{\mathrm{a}}$ & 0,004 \\
\hline Brincadeira simbólica & $3(3-3)^{b}$ & $1(1-1)^{\mathrm{a}}$ & $1,5(1-3)^{\mathrm{a}}$ & $<0,001$ \\
\hline Sequência de tópicos (brincadeira simbólica) & - & $3(2-3)$ & $3(1,8-3)$ & 0,761 \\
\hline Qualidade representação & - & $3(2-3)$ & $2(2-3)$ & 0,414 \\
\hline (Brincadeira Simbólica) & $2(1-2)$ & $1(1-1)$ & $1(1-1)$ & $\mathbf{0 , 0 0 2}{ }^{\dagger}$ \\
\hline Movimentos das mãos & $2(1-2)^{b}$ & $1(1-1)^{\mathrm{a}}$ & $1(1-1)^{\mathrm{a}}$ & $<0,001$ \\
\hline
\end{tabular}

Nota. ${ }^{*}$ Teste de Kruskal-Wallis. a,b Letras iguais (e.g.: a, a) não diferem pelo teste de Mann-Whitney ajustado por Finner. $\dagger$ O ajuste por Finner não pode identificar as diferenças significativas entre os grupos apontadas pelo teste de Kruskal-Wallis.

2 Para o autor, valores menores que 0,20 representariam uma concordância pobre, valores entre 0,21 e 0,40 fraca, entre 0,41 e 0,60 moderada, 0,61 a 0,80 boa e 0,81 a 1,00 uma concordância muito boa. 
as diferenças foram significativas serão apresentados neste estudo (ver Marques, 2011 para maiores detalhes).

Quando analisada a associação entre os diferentes grupos e as respostas para cada item do protocolo, verificou-se que, em geral, o grupo AU apresentou significativamente maior porcentagem de respostas nos escores 2 ou 3, apontando para maior comprometimento na maioria das variáveis, quando comparado aos demais grupos. As associações mais significativas $(p \leq 0,001)$ foram encontradas nos itens: Atenção Compartilhada (teste qui-quadrado de Pearson; $\chi^{2}$ $=18,9 ; g l=4 ; p=0,001$ ), Brincadeira Simbólica (teste quiquadrado de Pearson; $\left.\chi^{2}=19,1 ; g l=4 ; p=0,001\right)$ e Movimentos Estereotipados do Corpo (teste qui-quadrado de Pearson; $\chi^{2}$ $=18,3 ; g l=2 ; p<0,001)$, conforme apresentado na Tabela 2 . baixa intensidade. Todas as crianças (100\%) dos grupos SD e DT apresentaram o escore 1 , que significa ausência de movimentos repetitivos.

\section{Discussão}

No que se refere às evidências de validade de critério, buscou-se verificar quais itens diferenciavam melhor o grupo com AU dos controles, considerando a hipótese de que os direcionados à avaliação da Atenção Compartilhada, Imitação Espontânea, Brincadeira Simbólica e Comportamentos Repetitivos indicariam maior comprometimento nas crianças

Tabela 2. Frequências e Porcentagens Resposta em cada Item por Grupo

\begin{tabular}{lccc}
\hline \multicolumn{1}{c}{ Variáveis/Item } & $\begin{array}{c}\text { Grupo AU } \\
\mathrm{n}(\%)\end{array}$ & $\begin{array}{c}\text { Grupo SD } \\
\mathrm{n}(\%)\end{array}$ & $\begin{array}{c}\text { Grupo DT } \\
\mathrm{n}(\%)\end{array}$ \\
\hline Atenção compartilhada & & & $\boldsymbol{p}^{*}$ \\
1 & $2(20,0)$ & $9(90,0)$ & $10(100,0)^{* *}$ \\
2 & $3(30,0)$ & $1(10,0)$ & $0(0,0)$ \\
3 & $5(50,0)^{* *}$ & $0(0,0)$ & $0(0,0)$ \\
Brincadeira simbólica & & & $5(50,0)$ \\
1 & $0(0,0)$ & $9(90,0)^{* *}$ & $1(10,0)$ \\
2 & $0(0,0)$ & $0(0,0)$ & $4(40,0)$ \\
3 & $10(100,0)^{* *}$ & $1(10,0)$ & $10(100,0)^{* *}$ \\
1 & & $10(100,0) * *$ & 0,001 \\
2 & $3(30,0)$ & $0(0,0)$ & $0,0)$ \\
\hline
\end{tabular}

Nota. A opção de resposta 8 refere-se à má qualidade da imagem no vídeo, não permitindo uma análise adequada do item. *Teste quiquadrado de Pearson. ** Associação estatisticamente significativa pelo teste dos resíduos ajustados $(\mathrm{p}<0,05)$.

Em relação ao item Atenção Compartilhada, $50 \%$ das crianças do grupo AU obtiveram escore 3, significando que metade das crianças neste grupo não apresentou gestos/ verbalizações coordenados com olhar de forma frequente e dirigidos a uma variedade de situações, ao passo que nenhum dos participantes dos grupos SD e DT apresentou este escore. Por outro lado, todos os participantes do grupo DT $(100 \%)$ apresentaram escore 1 , ou seja, presença do comportamento de atenção compartilhada dirigida a uma variedade de situações.

Ao ser analisado o item Brincadeira Simbólica, todas as crianças do grupo AU não apresentaram esta habilidade (100\% no escore 3). O grupo SD foi o que obteve o melhor escore (escore 1), com 90\% das crianças apresentando brincadeira simbólica centrada ou transcendendo o próprio corpo (e.g., alimentar ou dar banho na boneca), embora ainda restrita às experiências domésticas (situações de alimentação, higiene etc.). O grupo DT não apresentou associação significativa com nenhuma das categorias de resposta.

Sobre os Movimentos Estereotipados do Corpo (mas não das mãos), a maioria das crianças (70\%) do grupo $\mathrm{AU}$ obtiveram escore 2 , apresentando movimentos repetitivos e involuntários ocasionais ou frequentes, mas sempre de do primeiro grupo. Com exceção do item Imitação, os resultados confirmaram a hipótese inicial do estudo.

Sobre a habilidade de Atenção Compartilhada, os resultados demonstraram que o desempenho das crianças com AU diferenciou-as dos grupos controle. O grupo com AU teve o pior desempenho tanto para iniciar (item 2) quanto para responder (item 4) às iniciativas dos adultos de compartilhamento da atenção sobre um objeto/evento. Esse achado é consistente com a literatura, que aponta a AC como principal marcador de desenvolvimento e preditor das habilidades sociais, comunicativas e linguísticas nas crianças com AU em idade pré-escolar (Smith, Mirenda, \& Zadman-Zait, 2007).

Mais especificamente, a habilidade em iniciar espontaneamente comportamentos de AC parece estar relacionada ao desenvolvimento sociocomunicativo global, subsequentemente (Delinicolas \& Young, 2007). Por outro lado, a habilidade em responder parece ter uma associação mais robusta especificamente com o desenvolvimento linguístico posterior (Dawson et al., 2004; Delinicolas \& Young, 2007). Uma análise mais detalhada acerca da distribuição dos escores em ambos os itens de AC mostrou que metade das crianças do grupo com autismo não 
apresentou comportamento declarativo de iniciativa e, em contrapartida, menos de um terço não respondeu ao adulto quando convidados a engajarem-se em brincadeiras.

Embora as análises se refiram à $\mathrm{AC}$, estes resultados demonstram a importância da avaliação de ambos os comportamentos separadamente, pois a verificação da competência em um, não garantiria êxito no outro. Ademais, deve-se considerar a implicação deste resultado para decisões terapêuticas, em que se deve planejar o trabalho direcionado para o aprimoramento da iniciativa e da resposta, tanto em termos de qualidade quanto de frequência dos comportamentos.

A área do PRO-TEA direcionada para o julgamento da relação da criança com os objetos/brinquedos foi construída a partir de uma perspectiva desenvolvimental, ou seja, contemplando a sequência de mudanças observadas no desenvolvimento infantil para o brincar. As categorias da brincadeira consideradas incluem a exploratória, a funcional e a simbólica. Há evidências empíricas de que a brincadeira de crianças com AU seguiriam essa sequência, porém apresentariam limitações no que tange a sua frequência e variedade; além disso, seriam caracterizadas predominantemente pela brincadeira exploratória ou funcional, com restrições na qualidade simbólica (Blanc et al., 2005).

Em consonância com a literatura apresentada e com a hipótese inicial deste estudo, o item que avaliou a Brincadeira Simbólica diferenciou o grupo com AU dos controles, sendo que o desempenho nessa habilidade foi significativamente pior no primeiro grupo. Ao proceder à análise da performance das crianças do grupo AU por meio da distribuição dos escores nesse item, pode ser verificado que nenhuma das crianças deste grupo apresentou brincadeira simbólica, enquanto que boa parte apresentou brincadeira exploratória ou funcional.

Em contrapartida, 90\% das crianças do grupo SD e 50\% das crianças do grupo DT apresentou Brincadeira Simbólica, em que um objeto foi utilizado para representar o outro, embora tenha ficado restrito a situações que representassem rotinas domésticas (e.g.: brincar de fazer comida). $\mathrm{O}$ fato de $40 \%$ das crianças do grupo DT não ter apresentado este tipo de brincadeira deve-se a uma limitação na equiparação dos grupos. A média de idade situou-se ao redor dos dois anos, período em que a brincadeira simbólica começa a surgir de forma mais complexa.

De fato, a análise dos resultados nos mostra que $90 \%$ das crianças deste grupo se engajaram em brincadeiras com instrumentos sonoros (e.g.: xilofone), jogos de encaixe e aqueles análogos ao real (e.g.: telefone de plástico), ao invés do substituto (e.g.: lego representando um telefone). Já no grupo AU, apenas 20\% apresentaram Brincadeira Funcional frequente e variada, sendo que em $70 \%$, esta foi ocasional e $10 \%$ não apresentaram este tipo de brincadeira. A partir desses dados, pode-se constatar que a maioria crianças com autismo foi capaz de brincar compreendendo a função dos brinquedos (e.g.: encaixando objetos com uma finalidade apropriada), embora não de maneira frequente e variada.

A avaliação dos Comportamentos Repetitivos e Estereotipados no PRO-TEA demonstrou que o único item que diferenciou as crianças do grupo AU das do grupo SD e DT foi o que avaliou os Movimentos Repetidos do Corpo (e.g.: rodopiar, pular para cima e para baixo, balanço, flapping - agitar os braços para cima e para baixo, caminhar na ponta dos pés/calcanhar etc). Esses foram frequentes em $70 \%$ das crianças do grupo $\mathrm{AU}$, ao passo que nas crianças dos demais grupos não foi registrada nenhuma ocorrência.

Apesar de não terem sido encontradas diferenças significativas entre grupos no item que avaliou os Movimentos Repetidos das Mãos (também classificados como Comportamentos Repetitivos Simples), foi possível observar que estiveram associados ao grupo AU quando considerada sua frequência ocasional durante a observação. As estereotipias manuais não foram observadas nos grupos SD e DT.

Goldman et al. (2008) encontraram resultados similares ao estudarem quatro grupos clínicos (AU de alto funcionamento cognitivo, de baixo funcionamento cognitivo, distúrbio especifico de linguagem e deficiência mental). Os autores descreveram que as estereotipias manuais e outros movimentos estereotipados como os de correr e pular, estiveram mais associados aos grupos com autismo, embora não restritos a este grupo, resultados consistentes com outros estudos (Honey, Leekam, Turner, \& McConachie, 2007; Matson, Dempsey, \& Fodstad, 2009).

Por outro lado, alguns estudos apontaram que somente os Comportamentos Repetitivos Complexos (CRC) estariam reservados ao TEA (Moraes, 2004). Os CRC, caracterizados por interesses restritos, linguagem repetitiva (ecolalia imediata e tardia), insistência na mesmice e presença de rotinas e rituais altamente elaborados estendidos às atividades de outras pessoas, seriam difíceis de serem detectados nas avaliações observacionais em laboratório como as deste estudo, pois estariam atrelados a contextos relacionados a atividades de rotina da criança (Honey et al., 2007).

Ao contrário dos demais comportamentos indicados na hipótese inicial deste estudo, na habilidade de Imitação os resultados encontrados não distinguiram o grupo AU dos controles. Sugeriu-se, inicialmente, que a imitação espontânea estaria mais prejudicada nas crianças com AU, posto que o PRO-TEA tem como característica a observação do comportamento sem a utilização de estratégias pelo avaliador para eliciar a imitação. Neste contexto espontâneo, os resultados mostraram que todas as crianças do grupo AU foram capazes de copiar gestos e/ou atividades iniciadas pela mãe, mas o fizeram apenas de forma ocasional, ao contrário dos demais grupos, nos quais estes ocorreram com maior frequência.

Em razão das respostas ao item contemplarem somente a frequência da imitação, pode-se pensar que esse fator, isoladamente, não tenha sido suficiente para distinguir os grupos. De fato, os estudos que afirmaram haver dificuldade de imitação nas crianças com TEA consideraram a frequência na análise dos resultados, mas adicionalmente focalizaram habilidades de imitação variadas (motoras gestuais ou dinâmicas, isoladas ou sequenciais, vocais, com ou sem objetos, de ações simbólicas ou não-simbólicas e imediatas ou diferidas).

Cabe ressaltar ainda que, nestes estudos, a situação experimental foi estruturada, ao contrário do presente estudo, em que o contexto foi livre (Colombi et al., 2009; McDuffie 
et al., 2007; Thurm, Lord, Lee, \& Newschaffer, 2006; Toth, Munson, Meltzoff, \& Dawson, 2006; Vanvuchelen, Roeyers, \& Weerdt, 2007). Na situação estruturada de imitação, o comportamento do avaliador estaria descontextualizado porque seria apresentado como uma "tarefa", o que demandaria maior compreensão acerca de suas intenções por parte da criança. No contexto livre, ao contrário, a imitação faria parte da brincadeira, de onde depreende-se que a compreensão do seu significado possa ser facilitada. Essa hipótese levantaria uma questão a ser investigada em futuros estudos.

De qualquer modo, permanece a dúvida: se a ausência de diferenças significativas deve-se a este fator ou a outros, como problemas na definição operacional do construto, por exemplo. Assim, sugere-se que esta última seja refinada a fim de se definir melhor os tipos de imitação. Uma hipótese alternativa de cunho conceitual, fundamentada em Tomasello (2003), distingue a aprendizagem por emulação de aprendizagem por imitação. A primeira focalizaria as mudanças de estado do meio provocadas pelo outro (e.g.: ver que um objeto começou a se movimentar), enquanto que a segunda trataria da estratégia comportamental do outro (dar corda no brinquedo), isto é, de suas intenções. $\mathrm{Na}$ primeira, os métodos comportamentais para atingir os objetivos são considerados apenas outros movimentos físicos do objeto e, portanto, indissociados do próprio objeto. Nesse sentido, pode ser que o item avalie apenas a aprendizagem por emulação que, teoricamente, estaria mais preservada em crianças com autismo, o que de qualquer forma aponta para a necessidade de refinamento operacional do item.

O exame dos itens também revelou outras limitações, como por exemplo, o reduzido intervalo da escala $(1,2$ e 3$)$, que acarretou pouca variação na distribuição dos escores. Outra implicação disto foi a amplitude da descrição em cada item, tornando-o ambíguo, já que nem sempre foi possível identificar qual parte da descrição se aplica a um determinado caso. Os itens da brincadeira ilustram esta dificuldade. Por exemplo, no item 18 (Brincadeira Simbólica), o escore 1 é descrito como "brinquedo centrado no próprio corpo - faz de conta que toma líquidos de uma xícara, come com talheres, penteia-se etc., fora do contexto de imitação das atividades do adulto; brinquedo transcende o próprio corpo, mas ainda centra-se em experiências domésticas - alimenta/penteia uma boneca, coloca-a para dormir; brinquedo transcende o próprio corpo e também as experiências domésticas médico/enfermeira, professora, loja etc.". Neste caso, apesar de haver representação da forma de usar um objeto em substituição a outro, não há uma distinção entre brincadeiras que transcendem ou não o próprio corpo, como é o caso de "papéis" (médico, papai/mamãe, professor etc.), sendo o último cognitivamente e socialmente superior ao primeiro (Tomasello et al., 2005).

Outro aspecto é que a tentativa de contemplar frequência e intensidade em um mesmo item parece ter contribuído para os problemas de ambiguidade. A utilização de escalas separadas para estas duas dimensões talvez minimize este problema. Por fim, o tempo empregado na aferição do protocolo também mostrou-se relativamente longo, se considerado o eventual uso em saúde pública. Isso aponta para a necessidade de uma possível redução do número de itens, com base nos achados do presente estudo.

\section{Conclusão}

O PRO-TEA constitui-se como uma medida potencialmente válida para a avaliação de crianças com suspeita de autismo, considerando-se que alguns dos seus itens foram capazes de discriminar crianças com autismo dos controles, mesmo em uma amostra reduzida. Sendo de custo relativamente baixo, se comparado a medidas internacionais, este instrumento poderá ser útil nos serviços públicos de saúde. Por se tratar de uma medida observacional e direta, o instrumento complementa outros como questionários $\mathrm{e}$ entrevistas, baseados em informações obtidas com os pais/ cuidadores.

Algumas limitações deste estudo apontam para sugestões de futuras pesquisas. Primeiro, o tamanho da amostra foi reduzido, embora seja comparável com outras pesquisas que têm examinado o desenvolvimento de crianças com esse diagnóstico no Brasil como, por exemplo, o de Miilher e Fernandes (2009). Além disso, o pareamento dos grupos foi realizado não por uma medida psicométrica, mas por meio de uma medida do vocabulário, baseada na informação dos pais. Pela necessidade de se trabalhar com algum parâmetro, considerou-se a forte associação que há entre vocabulário e atenção compartilhada (Smith et al., 2007).

É recomendável o pareamento por medidas mais rigorosas de nível cognitivo e de linguagem, incluindo as de QI, porém no momento da coleta de dados deste estudo não havia instrumentos validados e atuais, no Brasil, para a população pré-escolar, que fossem adequados para crianças com TEA. Por fim, os dados foram coletados a partir da interação com a mãe e não com um examinador não familiar à criança. É sabido que o comportamento de pais de crianças com autismo pode ser mais intrusivo e diretivo em função das peculiaridades da criança (Siller \& Sigman, 2002), o que poderia ter permitido ou não que a mesma manifestasse suas habilidades sociocomunicativas.

É importante estender tais achados com amostras maiores, grupos-controle com outras características clínicas (e.g.: distúrbio específico de linguagem), grupos com faixas etárias mais restritas (e.g.: dos 12 aos 24 meses), observação de contextos de interação com profissionais, ao invés dos pais e, por fim, o exame da readequação dos itens. Além disso, também são necessários estudos que utilizem outros instrumentos psicometricamente mais sofisticados e reconhecidos internacionalmente, no intuito de verificar outras evidências de validade deste instrumento, como a convergência de resultados em medidas que se propõe ao mesmo objetivo, além de cálculos de pontos de corte, exame de sensibilidade e especificidade. Neste exato momento, parte desta tarefa já está sendo realizada, demonstrando os desdobramentos do presente estudo, cujo foco é o refinamento dos itens, redução no tempo de administração e exame de propriedades psicométricas adicionais. 


\section{Referências}

Altman, D. G. (1991). Practical statistics for medical research. London: Chapman and Hall.

American Psychiatric Association. (2002). Manual diagnóstico e estatístico de transtornos mentais (C. Dornelles, Trans., $4^{\mathrm{a}}$ ed.). Porto Alegre: Artes Médicas.

American Psychiatric Association. (2013). Diagnostic and Statistical Manual of Mental Disorders ( $5^{\mathrm{a}} \mathrm{ed}$.). Arlington, VA: American Psychiatric Publishing.

Assumpção Jr, F. B., Kuczynski, E., Gabriel, M. R., \& Rocca, C. C. (1999). Escala de Avaliação de Traços Autísticos (ATA): Validade e confiabilidade de uma escala para detecção de condutas autísticas. Arquivos de Neuropsiquatria, 57(1), 23-29.

Ballabriga, M. C., Escudé, R. M., \& Llaberia, E. D. (1994). Escala d'avaluació dels trests autistes (ATA): Validez y fiabilidad de una escala para el examen de las conductas autistas. Revista de Psiquiatria Infanto-Juvenil, 4, 254-263.

Berument, S. K., Rutter, M., Lord, C., Pickles, A., \& Bailey, A. (1999). Autism Screening Questionnaire: Diagnosticvalidity. British Journal of Psychiatry, 175, 444-451.

Blanc, R., Adrien, J. L., Roux, S., \& Bartélémy, C. (2005). Dysregulation of pretend play and communication development in children with autism. Autism, 9(3), 229-245.

Bosa, C. (1998). Affect, social communication and selfstimulation in children with and without autism: A systematic observation study of requesting behaviours and joint attention. (Unpublished doctoral dissertation). Universidade de Londres, Inglaterra.

Castro-Souza, R. M. (2011). Adaptação Brasileirado M-CHAT (Modified Checklist for Autism in Toddlers) (Unpublished master's thesis). Universidade de Brasília, Brasília.

Centers for Disease Control and Prevention (2012). Prevalence of autism spectrum disorders: Autism and developmental disabilities monitoring network. Morbidity and Mortality Weekly Report, 61(3), 1-19.

Charman, T. (1997). The relationship between joint attention and pretend play in autism. Development and Psychopathology, 6, 249-261.

Charman, T. (2003). Why is joint attention a pivotal skill in autism? Philosophical Transactions of the Royal Society: Biological Sciences, 358(1430), 315-324.

Colombi, C., Liebal, K., Tomasello, M., Young, G., Warneken, F., \& Rogers, S. J. (2009). Examining correlates of cooperation in autism. Autism, 13(2), 143-163.

Dawson, G., Toth, K., Abbott, R., Osterling, J., Munson, J., \& Estes, A. (2004). Defining the early social attention impairments in autism: Social orienting, joint attention, and responses to emotions. Developmental Psychology, 40, 271-283.

Delinicolas, E., \& Young, R. (2007). Joint attention, language, social relating, and stereotypical behaviors in children with autistic disorder. Autism, 11(5), 425-436.

Di Napoli, F. O., \& Bosa, C. (2005). As relações entre a qualidade da interação mãe-criança e o reconhecimento da imagem de si em crianças com autismo. Revista Brasileira de Crescimento e Desenvolvimento Humano, 15(3), 11-25.

Goldman, S., Wang, C., Salgado, M. W., Greene, P. E., Kim, M., \& Rapin, I. (2008). Motor stereotypies in children with autism and other developmental disorders. Developmental Medicine and Child Neurology, 51, 30-38.
Honey, E., Leekam, S., Turner, M., \& McConachie, M. (2007). Repetitive behavior and play in typically developing children and children with autism spectrum disorders. Journal of Autism and Developmental Disorders, 37, 1107-1115.

Klin, A. (2006). Autismo e síndrome de Asperger: Uma visão geral. Revista Brasileira de Psiquiatria, 28(Supp.1), 3-11.

Krug, D. A., Arick, J. R., \& Almond, P. (1993). Behavior checklist for identifying severely handicapped individuals with high levels of autistic behavior. Journal of Child Psychology and Psychiatry, 21(3), 221-229.

Lifter, K. (2009). Developmental play assessment and teaching. In J. K. Luiselli, D. C. Russo, W. P. Christian \& S. M. Wilczynski (Eds.), Effective practices for children with autism: Educational and behavioral support interventions that work (pp. 299-322). Oxford University Press.

Lord, C., Rutter, M., Dilavore, P., \& Risi, S. (1999). Autism Diagnostic Observation Schedule. Los Angeles, CA: Western Psychological Services.

Lord, C., Rutter, M., \& Le Couteur, A. (1994). Autism Diagnostic Interview-Revised: A revised version of a diagnostic interview for caregivers of individuals with possible pervasive developmental disorders. Journal of Autism and Developmental Disorders, 24(5), 659-685.

Losapio, M. F., \& Pondé, M. P. (2008). Translation into portuguese of the M-CHAT Scale for early screening of autism. Revista de Psiquiatria do Rio Grande do Sul, 30(3), 221-229.

Marques, D. F. (2011). Aprimoramento e evidências de validade do protocolo de observação para crianças com suspeita de transtornos do espectro autista: um estudo preliminar (Unpublished master's thesis). Universidade Federal do Rio Grande do Sul, Porto Alegre.

Marteleto, M. R. F., \& Pedremônico, M. R. M. (2005). Validity of Autism Behavior Checklist (ABC): preliminary study. Revista Brasileira de Psiquiatria, 27(4), 295-301.

Matson, J. L., Dempsey, T., \& Fodstad, J. C. (2009). Stereotypies and repetitive/restrictive behaviours in infants with autism and pervasive developmental disorder. Developmental Neurorehabilitation, 12(3), 122-127.

McDuffie, A., Turner, L., Stone, W., Yoder, P., Wolery, M., \& Ulman, T. (2007). Developmental correlates of different types of motor imitation in young children with autism spectrum disorders. Journal of Autism and Developmental Disorders, 37, 401-412.

Meltzoff, A. N. (2005). Imitation and other minds: The "like me" hypothesis. In S. Hurley \& N. Chater (Eds.), Perspectives on imitation: From cognitive neuroscience to social science (pp. 55-77). Cambridge, MA: MIT Press.

Miilher, L. P., \& Fernandes, F. D. M. (2009). Habilidades pragmáticas, vocabulares e gramaticais em crianças com transtornos do espectro autístico. Pró-Fono Revista de Atualização Científica, 21(4), 309-314.

Moraes, C. (2004). Comportamentos repetitivos, interesses restritos e obsessões em indivíduos com transtorno global do desenvolvimento (Unpublished doctoral dissertation). Universidade Estadual de Campinas, São Paulo.

Mundy, P., \& Sigman, M. (1989). Specifying the nature of the social impairment in autism. In G. Dawson (Ed.), Autism: New perspectives on nature, diagnosis, and treatment (pp. 3-21). New York: Guilford. 
Pereira, A., Riesgo, R. S., \& Wagner, M. B. (2008). Autismo infantil: Tradução e validação da Childhood Autism Rating Scale para uso no Brasil. Jornal de Pediatria, 84(6), 487-494.

Rapin, I., \& Tuchman, R. F. (2009). Onde estamos: Visão geral e definições. In R. Tuchman \& I. Rapin (Eds.), Autismo: Abordagem Neurobiológica. Porto Alegre: Artmed.

Robins, D. L., Fein, D., Barton, M. L., \& Green, J. A. (2001). The modified checklist for autism in toddlers: An initial study investigating the early detection of autism and pervasive developmental disorders. Journal of Autism and Developmental Disorders, 31, 131-144.

Rutherford, M. D., Young, G. S., Hepburn, S., \& Rogers, S. J. (2007). A longitudinal study of pretend play in autism. Journal of Autism and Developmental Disorders, 37, 1024-1039.

Rutter, M., Le Couteur, A., \& Lord, C. (2003). Autism diagnostic interview revised manual. Los Angeles: Western Psychological Services.

Sanini, C. (2006). Comportamentos indicativos de apego em crianças com autismo (Unpublished master's thesis). Universidade Federal do Rio Grande do Sul, Porto Alegre.

Sato, F. P., Paula, C. S., Lowenthal, R., Nakano, E. Y., Brunoni, D., Schwartzman, J. S., \& Mercadante, M. T. (2009). Instrumento para rastreamento dos casos de transtorno invasivo do desenvolvimento - estudo preliminar de validação. Revista Brasileira de Psiquiatria, 31(1), 30-33

Schopler, E., Reichler, R., \& Renner, B. (1988). Childhood Autism Scale (CARS). Los Angeles: Western Psychological Services.
Sigman, M., Mundy, P., Sherman, T., \& Ungerer, J. (1986). Social interactions of autistic, mentally retarded and normal children. Journal of Child Psychology and Psychiatry, 27, 647-656.

Siller, M., \& Sigman, M. (2002). The behaviors of parents of children with autism predict the subsequent development of their children's communication. Journal of Autism and Developmental Disorders, 32(2), 77-89.

Smith, V., Mirenda, P., \& Zaidman-Zait, A. (2007). Predictors of expressive vocabulary growth in children with autism. Journal of Speech, Language, and Hearing Research, 50, 149-160.

Thurm, A., Lord, C., Lee, L. C., \& Newschaffer, C. (2006). Predictors of language acquisition in preschool children with autism spectrum disorders. Journal of Autism and Developmental Disorders, 37, 1721-1734.

Tomasello, M. (2003). Origens culturais da aquisição do conhecimento humano. São Paulo: Martins Fontes.

Tomasello, M., Carpenter, M., Call, T. B., \& Moll, H. (2005). Understanding and sharing intentions: The origins of cultural cognition. Behavioral and Brain Sciences, 28, 675-735.

Tomasello, M., \& Farrar, M. (1986). Joint attention and early language. Child Development, 57, 1454-1463.

Toth, K., Munson, J., Meltzoff, A. N., \& Dawson, G. (2006). Early predictors of communication development in young children with autism spectrum disorder: Joint attention, imitation, and toy play. Journal of Autism and Developmental Disorders, 36, 993-1005.

Vanvuchelen, M., Roeyers, H., \& Weerdt, W. (2007). Nature of motor imitation problems in school-aged boys with autism: A motor or a cognitive problem? Autism, 11(3), 225-240. 